\title{
Paranasal Sinus Involvement in Metastatic Carcinoma
}

\author{
Francois Abi-Fadel ${ }^{1}$ Peter R. Smith ${ }^{1}$ Asim Ayaz ${ }^{1}$ Krishnamurthi Sundaram ${ }^{2}$ \\ ${ }^{1}$ Division of Pulmonary Medicine, University Hospital of Brooklyn at \\ Long Island College Hospital, SUNY-Downstate Medical Center, \\ Brooklyn, New York, United States \\ 2 Department of Otolaryngology, University Hospital of Brooklyn at \\ Long Island College Hospital, SUNY-Downstate Medical Center, \\ Brooklyn, New York, United States

\begin{abstract}
Address for correspondence and reprint requests Peter R. Smith, M.D., Division of Pulmonary Medicine, University Hospital of Brooklyn at Long Island College Hospital, SUNY-Downstate Medical Center, 339 Hicks Street, Brooklyn, NY, 11201, United States

(e-mail: peter.smith@downstate.edu).
\end{abstract}

J Neurol Surg Rep 2012;73:57-59.

\section{Introduction}

Paranasal sinus metastases are uncommon. Since 1951 there have been 167 cases reported in the literature. The broad spectrum of signs and symptoms and rhinoscopic and imaging findings mimic many more common conditions, and the true etiology may be overlooked or missed for a considerable period. We describe a patient with known adenocarcinoma of the lung who was treated for recurrent sinusitis for 2 months before metastases to the paranasal sinus were identified. The results of an extensive review of the literature are presented.

\section{Case Report}

A 71-year-old man with adenocarcinoma of the lung was admitted with headaches, lethargy, fever, and facial swelling. His cancer diagnosed 1 year earlier was managed with chemotherapy (cis-platinum and paclitaxel), because advanced chronic obstructive lung disease precluded adequate resection. The medical history also included coronary artery disease with prior stent placement. He continued to smoke heavily and had abused alcohol in the past. Two months earlier he had been admitted to the intensive care unit (ICU)

received

January 13, 2012

accepted after revision

April 23, 2012

published online

August 8, 2012 and treated successfully for septic shock attributed to chronic sinusitis. Computed tomography (CT) at that time showed extensive sinusitis involving the sphenoid, ethmoid, and maxillary sinuses. Prominent soft tissue was seen in the posterior nasopharynx. A bone scan showed focal areas of increased activity compatible with metastatic disease in both maxillary sinuses, the right orbit, posterior midline skull, base of the skull, ribs, sternum, thoracic and lumbar vertebrae, pelvis, left ischium, and right proximal tibia. Further chemotherapy was planned.

On the current admission, the nasal mucosa was markedly edematous and necrotic debris was visible on the left side. There was periorbital edema and ptosis on the left. He again developed septic shock and the only identifiable source was again sinusitis. Rhinoscopy showed necrotic debris in the nose. Nasal cultures grew methicillin-sensitive Staphylococcus aureus and Enterococcus. Blood cultures were negative. He was managed in the ICU and improved with broad-spectrum antibiotic coverage. CT now showed complete opacification of the nasal cavities and all paranasal sinuses bilaterally. There was a soft tissue mass bulging into the left orbital cavity (-Fig. 1).
Copyright $\odot 2012$ by Thieme Medical Publishers, Inc., 333 Seventh Avenue, New York, NY 10001, USA. Tel: +1(212) 584-4662.
DOI http://dx.doi.org/ 10.1055/s-0032-1323157. ISSN 2193-6358. 


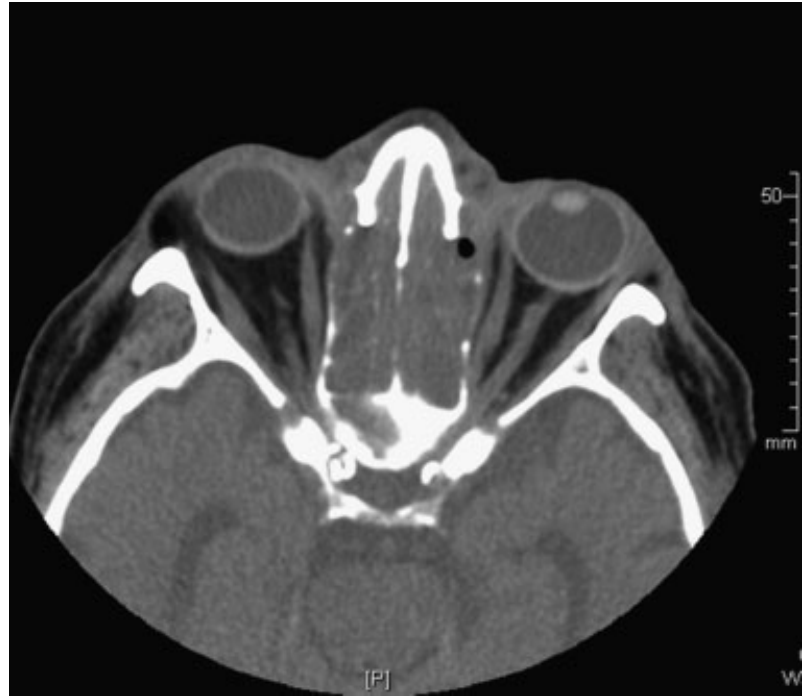

Figure 1 Opacification of ethmoid sinuses and soft tissue bulging into the left orbital cavity.

An orbital abscess was suspected, and there was concern about a possible fungal etiology with superimposed bacterial infection. As the nose was filled with soft tissue and necrotic debris, he underwent external ethmoidectomy. When the ethmoid was opened a tumor mass was found. Biopsy showed poorly differentiated adenocarcinoma histologically similar to the prior lung biopsies. Immunohistochemical staining confirmed metastatic adenocarcinoma of the lung. The patient expired several weeks later due to intracerebral hemorrhage.

\section{Discussion}

Solid tumor metastases involving the nasal cavities and paranasal sinuses are uncommon. A review of the literature from July 1951 to June 2011 yielded a total of 167 cases. Metastatic disease to the head and neck from distant sites is usually localized within the regional lymphatic chains. ${ }^{1}$ In a majority of cases metastatic disease involves only one of the paranasal sinuses, and the maxillary sinuses are the most frequent sites. ${ }^{2-4}$ In our patient, although metastatic tumor was documented only in the left ethmoid sinus, metastases to multiple sinuses were likely. The bone scan showed increased uptake in both maxillary sinuses and the degree of resolution obtained with this technique is often not sufficient to clearly separate uptake in adjacent structures. Moreover, the bone scan would not identify soft tissue metastases within the sinuses. Neither positron emission tomography (PET) scan nor postmortem examination was available to fully identify the extent of paranasal sinus involvement in our patient.

Renal carcinomas, the most common primary tumors that spread to the paranasal sinuses, probably do so via the vertebral vein plexus, ${ }^{5}$ which communicates with the great venous plexus of the head and the plexus of the paranasal sinuses. ${ }^{6}$ Hematogenous spread from occult lung metastases, and transcribrosal spread when there is meningeal involvement, are other possible routes. ${ }^{7}$

Of the 71 cases of renal cancer reported in the literature, clear cell carcinoma (hypernephroma), is by far the most frequent. Only 2 were adenocarcinomas. Breast cancer is the next most common primary accounting for 22 cases. Lung cancer was the third most frequent and prostate cancer the fourth, responsible for 17 and 16 cases, respectively. ${ }^{3,8-10}$ Thyroid carcinomas, hepatocellular carcinoma, colon, rectal, endometrial, bladder, fallopian tube, laryngeal, esophageal, gastric, pancreatic, and cervical carcinomas are each responsible for a few cases, as are melanomas, plasmacytomas, and Ewing sarcomas. Histologically, adenocarcinoma is the most commonly encountered cell type. ${ }^{2}$

Metastases to the paranasal sinuses present with a wide range of symptoms and physical findings. Headaches, visual disturbances, facial masses, facial pain, nasal swelling, nasal obstruction, and epistaxis are the most common presenting complaints. $^{2,8,11}$ Orbital symptoms may include proptosis, diplopia, lid edema, decreased vision, ptosis, blepharoptosis, and epiphora. Visual impairment is most often seen when metastases involve the sphenoid sinus because of its proximity to cranial nerves II, III, IV, and VI. ${ }^{2}$ Metastases to the frontal sinuses often present as a forehead mass. A patient with metastases to the frontal sinuses from bronchogenic carcinoma masquerading as a Pott puffy tumor has been reported. ${ }^{12}$ Maxillary sinus metastases present with epistaxis, facial pain, and proptosis. ${ }^{2}$

The differential diagnosis of paranasal sinus metastases includes Wegener's granulomatosis, midline granuloma, and primary neoplasms of the sinonasal tract. CT, magnetic resonance imaging, and angiography may show soft tissue masses involving the sinus cavities. Rhinoscopy may show a mass or bulging of soft tissues. Definitive diagnosis requires biopsy confirmation ${ }^{11}$ that may be obtained via endoscopic or open surgical procedures. Autopsy remains a not infrequent means of diagnosis since the true nature of nasal, sinus, or facial complaints may be missed antemortem.

Treatment of paranasal sinus metastatic disease in the majority of affected patients will be palliative only. $1,8,11,13$ Chemotherapy and/or radiation therapy are the usual options, although in selected cases surgical excision may be of benefit. ${ }^{2,8,14}$ Mean survival time is 5.7 months. However, paranasal sinus metastases due to renal carcinomas, the most frequent underlying primary, may be associated with 5-year survival rates as high as 15 to $30 \% .^{8,11}$

\section{Conclusion}

Metastases to the paranasal sinuses from distant primary carcinomas are unusual and often misdiagnosed. It behooves otolaryngologists to be familiar with this entity and consider this possibility in patients with nasal, sinus, or facial complaints who have a history of cancer, especially renal cancer. 


\section{References}

1 Kamiński B, Kobiorska-Nowak J, Bień S. [Distant metastases to nasal cavities and paranasal sinuses, from the organs outside the head and neck]. Otolaryngol Pol 2008;62:422-425

2 Huang CT, Hong RL. Nasion swelling as the presenting symptom of lung adenocarcinoma. J Thorac Oncol 2009;4:555-558

3 Prescher A, Brors D. [Metastases to the paranasal sinuses: case report and review of the literature]. Laryngorhinootologie 2001; 80:583-594

4 Bernstein JM, Montgomery WW, Balogh K Jr. Metastatic tumors to the maxilla, nose, and paranasal sinuses. Laryngoscope 1966; 76:621-650

5 Nanbu A, Tsukamoto T, Kumamoto Y, et al. Squamous cell carcinoma of bladder diverticulum with initial symptoms produced by metastasis to maxillary sinus. Eur Urol 1988;15:285-286

6 Sgouras ND, Gamatsi IE, Porfyris EA, et al. An unusual presentation of a metastatic hypernephroma to the frontonasal region. Ann Plast Surg 1995;34:653-656

7 Monserez D, Vlaminck S, Kuhweide R, Casselman J. Symmetrical ethmoidal metastases from ductal carcinoma of the breast, suggesting transcribrosal spread. Acta Otorhinolaryngol Belg 2001; $55: 251-257$
8 Torres Muros B, Bonilla Parrilla R, Solano Romero JR, Rodríguez Baró JG, Verge González J. [Metastasis in maxilar sinus as only manifestation of disseminate renal adenocarcinoma]. An Otorrinolaringol Ibero Am 2007;34:231-236

9 Argibay Vázquez S, Lancha Hernández C, Martínez Muñiz A. Metastases in the sphenoidal sinus in a patient with papillary thyroid cancer. Clin Transl Oncol 2005;7:324-327

10 Pereira Arias JG, Ullate Jaime V, Valcárcel Martín F, et al. [Epistaxis as initial manifestation of disseminated renal adenocarcinoma]. Actas Urol Esp 2002;26:361-365

11 Montoro Martínez V, López Vilas M, Gurri Freixa M, De Dios Orán E, Montserrat Gili JR, Fabra Llopis JM. [Nasal sinus metastasis of renal carcinoma. A case report]. Acta Otorrinolaringol Esp 1999;50:653656

12 Clarkson JH, Kirkland PM, Mady S. Bronchogenic metastasis involving the frontal sinus and masquerading as a Pott's puffy tumour: a diagnostic pitfall. Br J Oral Maxillofac Surg 2002;40:440-441

13 Traserra J, Morelló G, Traserra-Coderch J. [Metastasis of systemic origin in otorhinolaryngology]. Rev Neurol 2000;31:1265-1267

14 Simo R, Sykes AJ, Hargreaves SP, et al. Metastatic renal cell carcinoma to the nose and paranasal sinuses. Head Neck 2000;22:722-727 